\title{
Sensor-based Real-time Resource Model Reconciliation for Improved Mine Production Control - A Conceptual Framework
}

\author{
J Benndorf', M Buxton² and M S Shishvan³
}

\begin{abstract}
The flow of information, and consequently the decision-making along the chain of mining from exploration to beneficiation, typically occurs in a discontinuous fashion over long timespans. In addition, due to the uncertain nature of the knowledge about deposits and the inherent spatial distribution of material characteristics, actual production performance in terms of produced ore grades and quantity and extraction process efficiency often deviate from expectations. Reconciliation exercises to adjust mineral reserve models and planning assumptions are performed with timely lags of weeks, months or even years.

With the development of modern information and communication technology over the last decade, a flood of data about different aspects of the production process is available in a realtime manner. For example, sensor technology enables online characterisation of geochemical, mineralogical and physical material characteristics on conveyor belts or at working faces. The ability to utilise this additional information and feed it back into reserve block models and planning assumptions opens up new opportunities to continuously control the decisions made in production planning to increase resource recovery and process efficiency. This leads to a change in paradigm from a discontinuous method to near real-time reserve reconciliation and model updating, which calls for suitable modelling and optimisation methodologies to quantify prior knowledge in the reserve model, process and integrate information from different sensor sources, back propagate the gain in information into reserve models and efficiently optimise operational decisions in real-time.

This paper introduces the concept of an integrated closed-loop framework for real-time reserve management, incorporating sensor-based material characterisation, geostatistical modelling under uncertainty, modern data assimilation methods for sequential model updating and mining system simulation and optimisation. The effectiveness of the framework and the value added will be demonstrated in an illustrative case study.
\end{abstract}

\section{INTRODUCTION}

Successful planning and operations management in mineral resource extraction is based on a solid understanding of the spatial distribution of ore tonnages and grades in the deposit. Knowledge about the deposit is often based on exploration data and typically captured in a digital 3D resource model. Exploration data are gathered in campaigns prior to operation, which were often undertaken decades ago. The sample spacing is designed to capture major features of the deposit with the anticipated level of accuracy while minimising expenditure. Although resource models are created using sophisticated geostatistical modelling techniques, such as different types of kriging or conditional simulation (eg
Chiles and Delfiner, 2012), they can locally exhibit significant deviations from in situ resource characteristics.

Short-term production scheduling in mining operations is based on the resource model and aims to define an extraction sequence that meets short-term production targets in terms of ore tonnage produced and associated grades. The scale of short-term production targets can be as small as a train load in the order of $1000 \mathrm{t}$ that is shipped to the customer. However, such a scale is not supported by data gathered during exploration. The consequences can be unexpected deviations from production targets, which may have significant

1. GAusIMM, Assistant Professor, Resource Engineering, Department of Geosciences and Engineering, Delft University of Technology, Stevinweg $1,2628 \mathrm{CN}$ Delft, The Netherlands. Email: j.benndorf@tudelft.nl

2. Associate Professor, Resource Engineering, Department of Geosciences and Engineering, Delft University of Technology, Stevinweg 1, 2628CN Delft, The Netherlands. Email: m.w.n.buxton@tudelft.n|

3. PhD Candidate, Department of Geosciences and Engineering, Delft University of Technology, Stevinweg 1,2628CN Delft, The Netherlands. Email: m.soleymanishishvan@tudelft.nl 
economic impacts. Therefore, the understanding of shortscale variability of ore characteristics is critical to controlling the operation and meeting production targets.

As demonstrated in various case studies (eg Benndorf, 2009; Zimmer, 2012; Benndorf, 2013), short-scale variability and uncertainty in prediction can be modelled by conditional simulation and propagated through a transfer function to assess the expected performance of a short-term mine plan. Although this methodology allows the recognition of the magnitude and frequency of potential deviations, it does not lead to an increase in knowledge since no additional data are included in the decision-making process.

With the developments in information and communication technology over the past decade, online data capturing of production performance provides an alternative source of information. This means that a flood of real-time data is now available. Sensor technology for detecting the characteristics of raw materials on a conveyor belt has been proven in industrial field tests in some operations. Documented studies refer to the application of specific sensor technologies such as near-infrared (eg Goetz, Curtiss and Shiley, 2009) or dual energy X-ray transmission (Jong, Dalmijn and Kattentid, 2003). The application of sensors provides a high density of information on a short timescale with reasonable precision. The example in Figure 1 compares lab analyses and sensorbased measurements for coal ash content of train-car loads of approximately $100 \mathrm{t}$. The correlation coefficient of 0.93 suggests high information content of the sensor data.

To date, sensor information is mainly utilised in feed forward loops applied for downstream process control, such as supporting dispatch decisions, material sorting or blending on stockpiles (eg Scholze and Köhler, 2012; Sládková, Kapica and Vrubelm, 2011). An immediate feedback of sensor information into the resource and planning assumptions to continuously increase its certainty in prediction does not occur. However, the ability to feed data back suggests a significant potential for improvement in operational efficiency. With increased certainty of prediction of grades for reserve blocks, the frequency of misclassification and unfavourable dispatch decisions is expected to decrease. Buxton and Benndorf (2013) quantified this value in the order of $\$ 5 \mathrm{M} / \mathrm{a}$ for an averagesized operation. A breakthrough towards a 'self-learning mine' that utilises all available data for real-time feedback control and process optimisation requires fast integration and processing of data, a back-propagation of process information into the models and real-time decision support. A similar framework was recently developed in petroleum reservoir management (Jansen et al, 2009), demonstrating an increased process efficiency in the order of six to nine per cent.

This paper introduces a new and innovative framework for real-time reconciliation and optimisation for extractable reserves in continuous mining operations. It consists of a closed-loop approach (Figure 2), which feeds back sensor data into resource models and optimises operational decisions to account for the real-time information gained during production. First, the concept is described and the recent developments in the major pillars of the framework are documented. Then, selected aspects of the framework are demonstrated in an illustrative case study.

\section{MOVING TOWARDS REAL-TIME RESERVE MANAGEMENT - THE CLOSED LOOP CONCEPT}

Figure 3 illustrates the closed-loop concept for real-time reserve management (RTRM), which is defined by the following six steps:

1. Based on available exploration data, a resource model is generated and reserves are assessed as the basis for shortterm mine planning and production control. This model is referred to as a prior model. In general, techniques can involve geostatistical estimation as well as simulation.

2. Short-term mine planning and operational decisions are optimised to ensure that production targets are achieved most efficiently. These optimisation tasks may be performed using mathematical optimisation techniques such as mixed integer programming (MIP), stochastic programming or metaheuristic methods, or techniques of simulation-based optimisation for more complex systems.

3. Based on these optimised decisions and utilising the resource/reserve model, model-based expected process efficiency indicators and material characteristics can be predicted at different locations in the extraction and material handling process.

4. When executing the mine plan, sensor-derived measurements about the process efficiency and material quality can be taken at these different locations.

5. Differences between model-based prediction (step 3) and actual measurements (step 4) may have two different causes: a resource/reserve model error and a measurement error. Modern techniques of data assimilation are used to

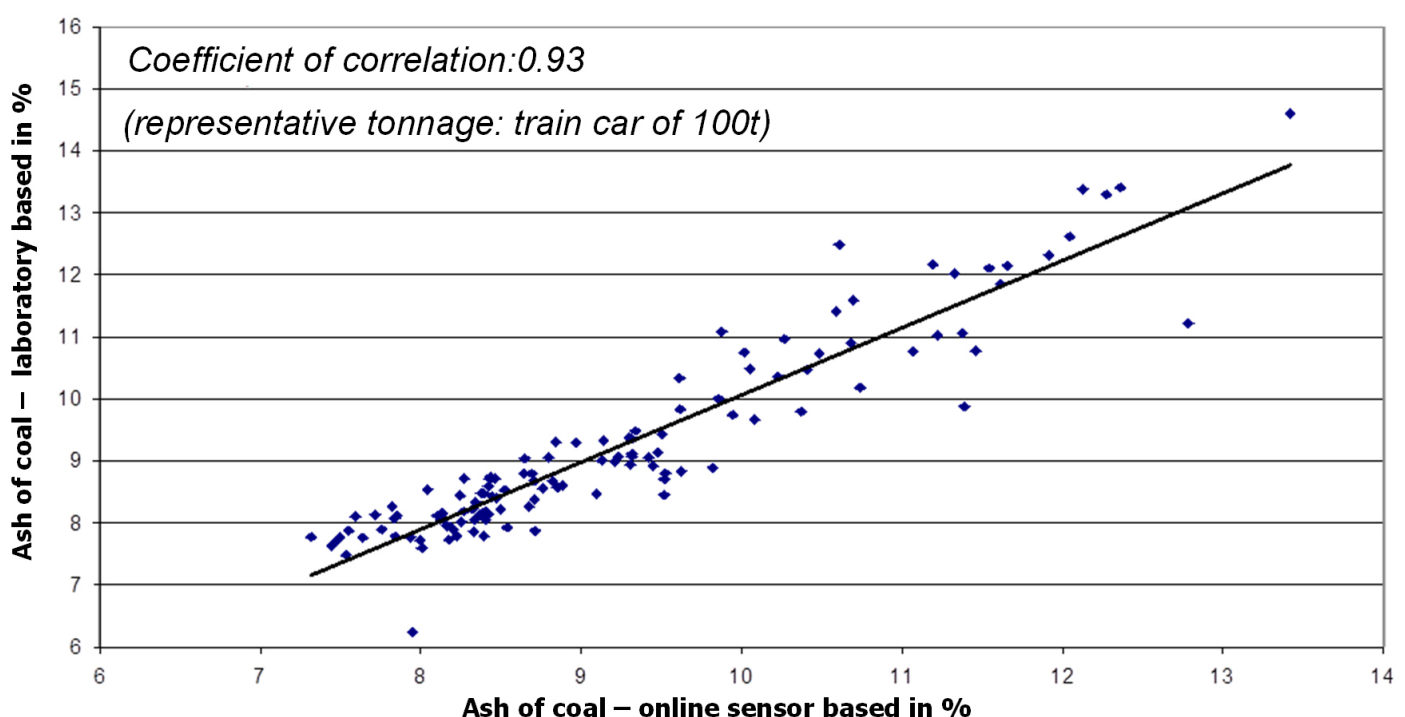

FIG 1 - Correlation between sensor-based measurements and lab analysis in coal samples. 


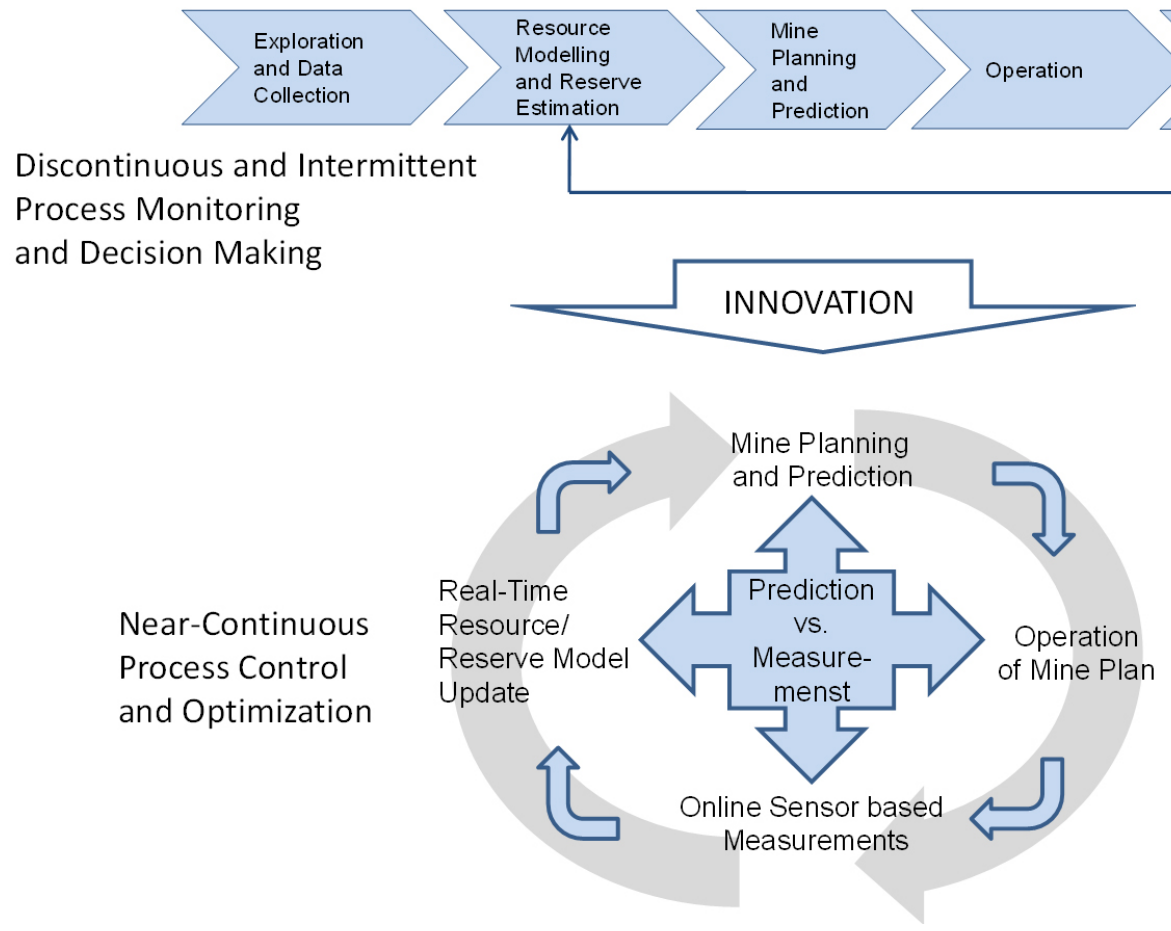

FIG 2 - Closed-loop concept for near-continuous process control and optimisation.

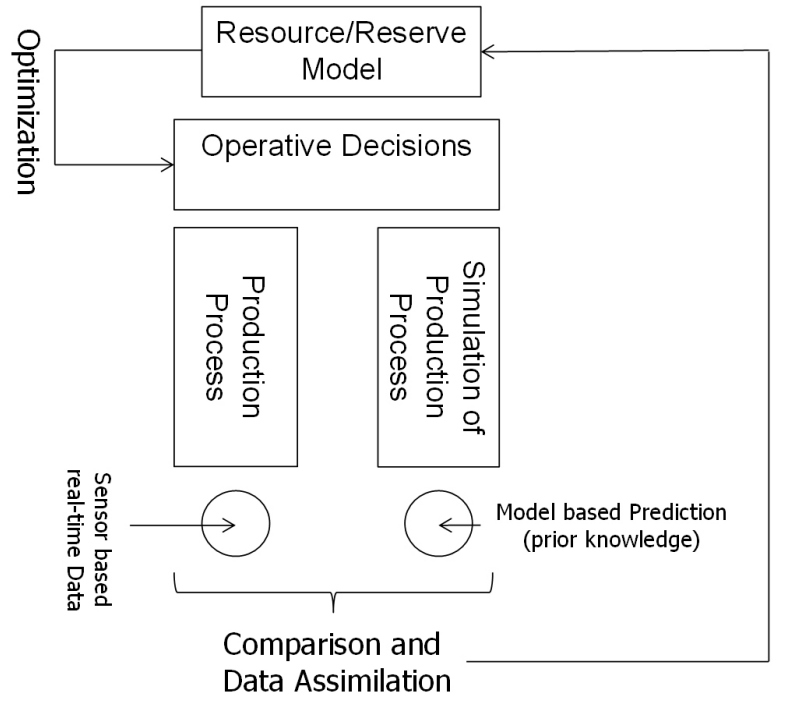

FIG 3 - Flow chart of real-time reserve management.

separate the influence of these two causes and utilise this information of the difference to update the prior resource/ reserve model (step 1) to obtain a posterior model.

6. Go back to step 2 and optimise short-term and operational decisions based on the updated posterior resource/ reserve model.

The proposed concept of RTRM is the subject of current research and technical development. Its maturation for industrial application requires further development in three main pillars:

1. sensor-based material characterisation

2. a real-time feedback loop of sensor data for resource/ reserve model updating

3. simulation-based optimisation for short-term production scheduling and production control.

The following three subsections provide an overview of recent developments in the areas of the three main pillars and propose algorithmic solutions and areas for future development.

\section{Online sensor-based raw material characterisation}

For real-time updating of the reserve model and mine optimisation, sensor-derived data are required to identify and discriminate raw material properties such as texture, mineralogy, geochemistry and physical properties prior to and during mining. Specific sensor techniques that have the potential to be used to satisfy these requirements include laser-induced breakdown spectroscopy (LIBS) (eg Death, Cunningham and Pollard, 2008), visible near-infrared (VisNIR) and short wave infrared (SWIR) imaging for determining textures and mineralogy; X-ray fluorescence (XRF) for geochemistry and thermal and mid-wave infrared and long wave infrared (LWIR) (eg Harris et al, 2010) for assessing silica content. Imaging techniques are required for size, volume and shape determination. These can contribute towards mass and density determination. The infrared (VisNir, SWIR and LWIR), XRF, Raman spectroscopy and LIBS methods require no pre-preparation of the sample.

Infrared spectral techniques can be used to determine mineralogical parameters for geological material through the use of different spectral ranges, including VisNIR (wavelength range 0.4-0.7 $\mu \mathrm{m}$ ) and SWIR (wavelength range 0.7-2.6 $\mu \mathrm{m}$ ). SWIR is an important rangefor providing mineralidentification for hydroxyl-, water- and carbonate-bearing minerals. Commercial applications are available, including airborne scanners, real-time assessment of materials on conveyor belts and monitoring of material during scheduling. Technologies such as the CSIRO HyLogger ${ }^{\mathrm{TM}}$ suite have been developed to provide voluminous and automated point data. These systems capture infrared data from drill core or chip samples. In a static mode, hyperspectral imaging systems for the logging of drill cores have been developed, such as the SisuRock system by Spectral Imaging Ltd in Finland. LWIR (wavelength range $6-14 \mu \mathrm{m}$ ) is one of the most important regions for mineralogy since direct detection and identification of rockforming silicates is possible. Hyperspectral LWIR imaging systems for mineral detection are commercially available for airborne scanners and static imagers. Applications for use 
in high-throughput environments have not yet been developed.

LIBS can be used for the analysis of solid, liquid and gaseous samples. An analysis can be performed in a few tenths of $\mu$ simultaneously for all chemical elements whose spectral lines lie in the detected spectral range of the spectrometer. Using modern data acquisition electronics, up to 1000 LIBS measurements per second are possible (Bette et al, 2005).

Mineral characterisation using Raman spectroscopy is well established. However, because Raman spectroscopy is a molecular technique, it is seldom used to characterise whole rocks such as those extracted during mining. With respect to Raman spectroscopy instrumentation, commercially available state-of-the art handheld instrumentation devices are designed for a specific task, which in most cases includes the pharmaceutical industry or homeland security. Although the hardware may be applicable to whole rock samples from mines, the software is not. Issues regarding resolution and optical quality in complex polymineralogical applications are not resolved.

Raman and LIBS can be combined to provide complementary detection solutions. The combination is attractive for remote mineralogical characterisation and has been increasingly studied by the National Aeronautics and Space Administration and the European Space Agency for lunar and Mars exploration (eg Sharma et al, 2003; Escudero-Sanz et $a l, 2008)$. LIBS shows high sensitivity in detecting cations and trace elements but is less sensitive in detecting anions. Raman can identify the anion groups in the crystals and crystal forms from Raman-active lattice modes (Sharma et al, 2007). LIBS/ Raman combinations also have potential for the mapping of heterogeneous minerals (Hoehse et al, 2009). However, there is a technology gap between the highly portable systems designed for space exploration, which are excessively expensive and highly overspecified for applications in a terrestrial industrial scenario, and the current state-of-theart bulky laboratory systems. A portable combined Raman and LIBS system for high-throughput mining applications does not currently exist, but is clearly required for practical measurements in an operational environment.

For all sensor types, imaging techniques may be required for size, volume and shape determination. These will contribute towards mass and density determination. Sensor resolution and the ability to discriminate differ for each of the different sensor types. Different sensor types generate different data outputs in terms of response, precision, accuracy and format. One specific sensor cannot satisfy all requirements.

There is no current application that integrates combinations of these sensors for comprehensive material characterisation and discrimination in a highly variable and large-throughput environment. Current research and development activities of such technology will enable real-time feedback loops for reserve updating.

\section{Real-time feedback loop for reserve model updating}

This part of the RTRM is designed as a back-propagation of process information into the resource/reserve model. To account for different data originating from different sources with a different data quality, density and support, the methods currently used in geostatistical modelling and data fusion have to be extended. Different data (eg from exploration holes and lab analysis, online responses of sensors, GPS measurements of actually mined raw material or geodetic survey data) have to be integrated consistently to update the reserve model in a Bayesian fashion. In addition, the material characterised at sensor locations may represent a blend of material originating from multiple phases and locations of extraction. In order to feed back the sensor information, the influence of material originating from each extraction face has to be separated.

To solve these challenges, multiple solutions are possible. This paper proposes a modification of Kalman filter (KF) techniques. These are designed to sequentially estimate the system states, in this case the local grades at excavation locations, recursively on the basis of noisy measured input data. Kalman (1960) introduced a method in the context of system and control theory describing a recursive solution to estimate the state of a stochastic process $Z^{t+1}$ at time $t+1$ based on a prior model of the state $Z^{t}$ at time $t$ and observations $l$ at time $t$.

To update a spatial resource model, the system state is put in a spatial context and represents the block model estimate $Z(x)$. The observations correspond to sensor measurements during a production period of a certain time span (eg five minutes or one hour).

The idea is to update the resource model, denoted with $Z^{t+1}(x)$ as a linear combination of the prior block model $Z^{t}(x)$ and the difference between model-based prediction and the vector of sensor-based measurements $l$ (Equation 1).

$Z^{t+1}(x)=Z^{t}(x)+K\left(l-A Z^{t}(x)\right)$

Matrix $A$ is a design matrix and captures the contribution of each reserve block per time interval to the raw material flow produced and observed at a sensor station. The term $A Z^{t}(x)$ represents the model-based prediction and integrates the operative decisions (digging capacity and location of excavators at each time) in $A$ and the prior resource/reserve model $Z^{t}(x)$. The objective is to determine the matrix $K$, which is the unknown updating factor (Kalman gain), as a best linear and unbiased estimator. A detailed derivation is out of the scope of this paper and the reader is referred to literature by Welsh and Bishop (1997) and Benndorf (in prep). It can be shown that:

$$
K=\left(A^{T} C_{Z Z}^{t} A+C_{l l}\right)^{-1} A^{T} C_{Z Z}^{t}
$$

An interpretation of Equation 2 reveals the integrative character of the Kalman gain. The first term is the inverse of two error sources:

1. the model prediction error, represented by the covariance matrix of the prior resource model $C_{Z Z}^{t}$, which is propagated through the mining system by the design matrix $A$

2. the measurement error, represented by the covariance matrix of the sensor-based measurement $C_{l l}$.

The second term again represents the error source of the model-based prediction. A comparison of potential magnitudes of the two error terms reveals that:

- If the model error is large and the measurement error small, the Kalman gain, $K$, tends towards one. The application to Equation 1 shows that the full difference between modelbased prediction and sensor-based measurement is taken into account to update the resource/reserve model.

- If the model error is small and the measurement error large, the Kalman gain, $K$, tends towards zero. The application to Equation 1 indicates that the difference between modelbased prediction and sensor-based measurement is not taken into account to update the resource/reserve model. The precision of the sensor is too low to add value to the estimation of resources and reserves. 
It is intuitive that with the integration of sensor data in the resource/reserve model, the prediction uncertainty decreases. This is not only the case for reserve blocks that are currently excavated, but also for adjacent blocks to be excavated because these are spatially correlated. It can be shown that the improvement in model prediction can be quantified by:

$C_{Z Z}^{t+1}=C_{Z Z}^{t}-K A C_{Z Z}^{t}$

where:

$C_{\mathrm{ZZ}}^{c+1}$ is the updated posterior model covariance matrix, which is by definition smaller than the prior model covariance matrix $C_{\mathrm{zz}}$.

Due to the storage and propagation of the error covariance matrix, KF-based approaches suffer from computational efficiency, especially when applied to large systems. To handle large problems with potential non-linear dynamics, the ensemble Kalman filter (EnKF) offers a solution (Evensen, 2003). Instead of propagating the covariance matrix in time using Equation 3, a finite set of so-called ensemble members is generated representing realisations $z(x)$ of the spatial random function $Z(x)$. Each ensemble member is an equally probable representation of the spatial random field at time $t$. The initial set of ensemble members can be generated using techniques of conditional simulation in geostatistics (eg Chiles and Delfiner, 2012). Using Equation 1, all ensemble members are propagated separately in time when new data $y$ are available (Figure 4).

Instead of storing the complete covariance matrix $C_{\mathrm{ZZ}}$, only a finite set of ensemble members is kept. If the number of ensemble members is sufficiently large, the covariance matrix can be approximated by:

$C_{\mathrm{ZZ}}^{t+1} \cong \frac{1}{N} \sum_{i=1}^{N}\left(z\left(x_{i}\right)-\overline{z(x)}\right) \cdot\left(z\left(x_{i}\right)-\overline{z(x)}\right)^{T}$

Note that in order to maintain the variance and covariance structure of the ensemble members, the observations have to be treated as random variables. For this reason, an error $\omega_{r}$ has to be added to the observations $l$ used to update the several ensemble members $r$ with $r=1, \ldots, N$.

\section{Simulation-based optimisation for short-term and operative decisions}

The updated model will possibly lead to new decisions in shortterm operation management such as production sequencing, digging capacity control or stockpile management. Methods of mathematical programming, such as dynamic programming or MIP, are well acknowledged in the field of mine planning optimisation (eg Ramazan and Dimitrakopoulos, 2004). Recently, research was successfully performed to integrate geological uncertainty (eg Dimitrakopoulos and Ramazan, 2009; Benndorf and Dimitrakopoulos, 2013), leading to an increase of 24 per cent in net present value while reducing the risk of not achieving production targets. Jewbali and Dimitrakopoulos (2009) introduced a short-term production scheduling optimisation based on geological uncertainty and updateable models, and demonstrated the benefit in the Australian gold mining industry. The aforementioned applications are small or moderate in size. Short-term production scheduling in large open pit mines represents a problem, which is typically complex and involves many interdependencies. These are difficult to model in a closed form.

Most of the mathematical programming approaches are limited by the amount of decision variables as applications become larger and suffer from reduced computational efficiency. In leading manufacturing process industries, such as the aerospace, chemical or petroleum engineering industries, the simulation approach is applied to support expensive decision-making and optimisation during the design and operation of processes (eg Young Jung et al, 2004; Schulze-Riegert and Shawket, 2007; Subramaniam and Gosavi, 2007). Simulation-based optimisation methods (Figure 5), such as response surface methods or learning automata search, have been proven to result in near optimal solutions for decision problems and are especially applicable for scheduling complex and computationally large systems (Gosavi, 2003), such as continuous mining operations. The concept of simulation-based optimisation is shown in Figure 5. Using general system simulation techniques, the objective value $J$ of a complex objective function can be evaluated for a given set of decision variables. The optimisation part, such as response surface methods in combination with gradient

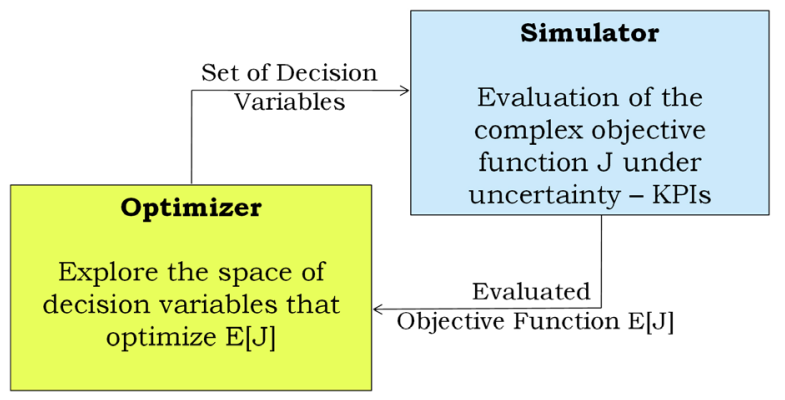

FIG 5 - The concept of simulation-based optimisation.

A non-linear Version: The Ensamble Kalman-Filter

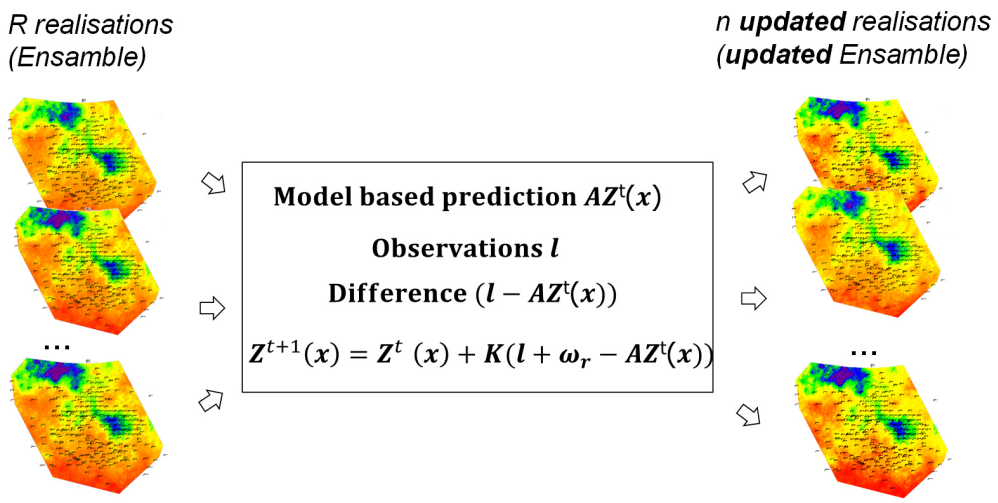

FIG 4 - The concept of the ensemble Kalman filter (reproduced after Evensen, 2003). 
descent methods, will explore the space of decision variables to obtain a near optimal set of these.

Stochastic process simulation, whether discrete, continuous or combined (Kelton and Law, 2000), provides a powerful tool for measuring performance indicators summarised in an objective function of complex systems. In essence, the simulator assesses a complex objective function $J$. Hall (2000) presented the requirement for successful simulation modelling, advantages and disadvantages of simulation as well as pitfalls for mining-related application in two case studies. The results showed that simulation can be a powerful tool for the mining engineer that, when used in proper applications, is able to provide insights into complex system behaviour. Baafi and Ataeepour (1996) and Askari-Nasab et al (2012) used discrete event simulation to investigate a truckshovel system of discontinuous open pit mines. The process simulation method was used to optimise the truck fleet size for the system. For short-term mine planning, Shishvan and Benndorf (2013) presented a simulation-based approach for continuous mining applications integrating geological uncertainty. The objective was to evaluate the performance in terms of producing the target quantity and quality in a large open pit coal operation and assess the efficiency for alternative production schedules. Different sets of decision variables were tested, including a shift schedule, block sequencing and defined production rates. Results demonstrated that the stochastic approach provides the mine planning engineer with a valuable tool to foresee critical situations affecting the continuous supply of raw material to the customers and system performance. Comparing the outcome of different sets of decisions provides a tool for improved decision-making.

Having powerful simulation tools for mining systems enables the impact of a set of short-term or control decision variables to be evaluated. Simulation-based optimisation is based on an iterative perturbation of decision variables and the mapping of the corresponding objective value $J$. Utilising the response surface method, the objective value can be mapped as a function of decision variables, even if not all possible combinations are tested. The efficient exploration of combinations of decision variables can be supported by stochastic gradient descent methods. The maximum of the resulting response surface of the objective values leads to optimal decision variables. Salama, Nehring and Greberg (2013) used a combination of discrete event simulation and MIP to improve decision-making in underground mining. The proposed method used the simulation approach to evaluate the operating costs of a set of different haulage system scenarios and obtained the cash flows for input into the MIP model.

Further work is required to extend current applications to continuous production control variables, such as effective digging rates, and include the short-term sequencing problem in the optimisation phase.

\section{AN ILLUSTRATIVE EXAMPLE FOR MODEL UPDATING}

The subsequent example investigates the performance of the proposed updating methodology for different mining system configurations and sensor precisions. For deeper insights into the continuous mine system simulation for short-term planning and decision control under geological uncertainty, the reader is referred to Shishvan and Benndorf (2013). Here, an artificial test case is presented, which is built around the well-known and fully understood Walker Lake data set (Isaaks and Srivastava, 1989). The data set (Figure 6) is interpreted as a quality parameter of a coal deposit (eg as calorific value). It is sampled irregularly at a spacing corresponding to an average of two reserve block lengths. The blocks were defined with a dimension of $16 \mathrm{~m} \times 16 \mathrm{~m} \times 10 \mathrm{~m}$. The block variogram is given with a spherical structure, range $50 \mathrm{~m}$, nugget effect 0.4 and sill 0.6 .

With an assumed density of $2 \mathrm{t} / \mathrm{m}^{3}$, one mining block represents a tonnage of $5.120 \mathrm{t}$. Ordinary kriging was used to generate a resource block model and the prior error covariance matrix, generalised sequential Gaussian simulation was used to derive the realisations or ensemble members for the EnKF application. For simplicity, no dilution and losses were applied, resulting in the reserve model being equal to the resource model. The resulting block model (Figure 6) was used as the prior model.

Without loss of generality, the artificial block model will be mined with a continuous mining system, which consists initially of two bucket wheel excavators positioned at separate benches (Figure 7). Figure 6 shows the extraction sequence for the case of two excavators. Different digging rates were applied: excavator one mines at a rate of $500 \mathrm{t} / \mathrm{h}$ and excavator two at $1000 \mathrm{t} / \mathrm{h}$. The material is discharged on belt conveyors positioned on the benches, which are combined to one material flow at the central mass distribution point. The belt speed is assumed to be constant at $6 \mathrm{~m} / \mathrm{s}$.

The combined material flow of both excavators is scanned by a sensor positioned above a central conveyor feeding the stock and blending yard. Since no real sensor data were available, virtual sensor data were generated. The
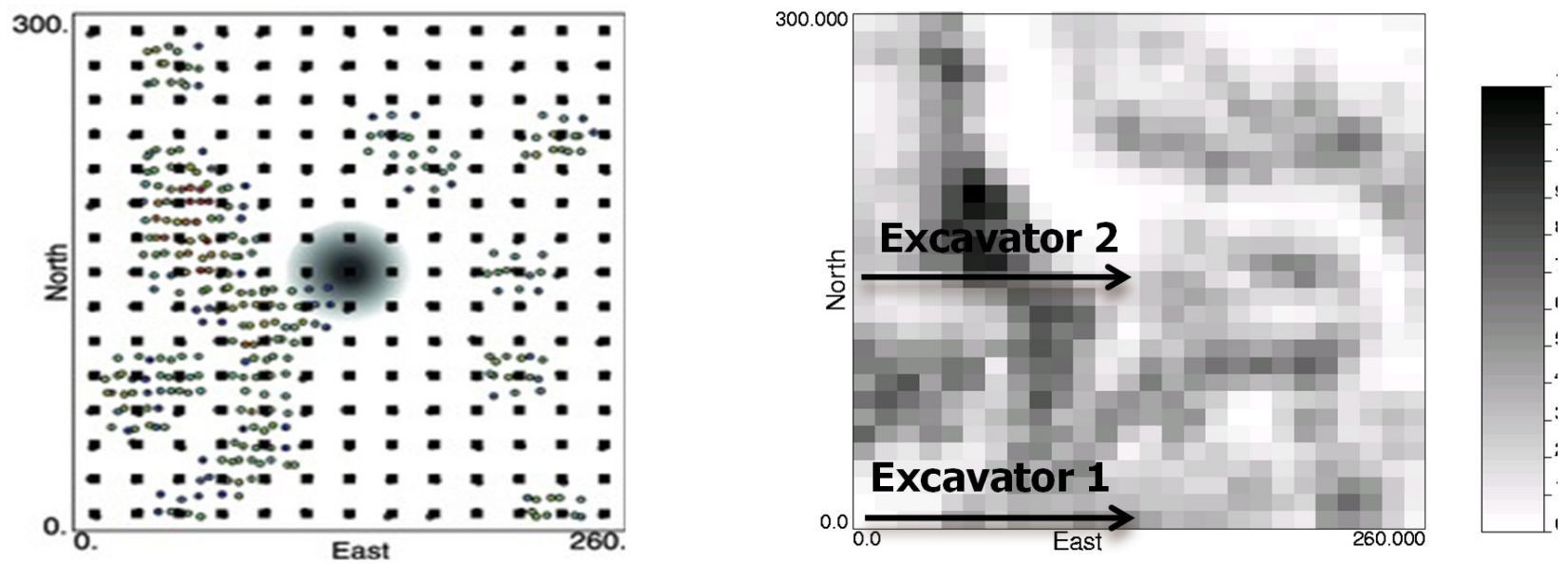

12.0

$11.0 \stackrel{9}{9}$

10.0 을

9.0 京

8.0

$7.0 \stackrel{\overrightarrow{\hat{\theta}}}{ }$

6.0

FIG 6 - Set-up for illustrative case study. 


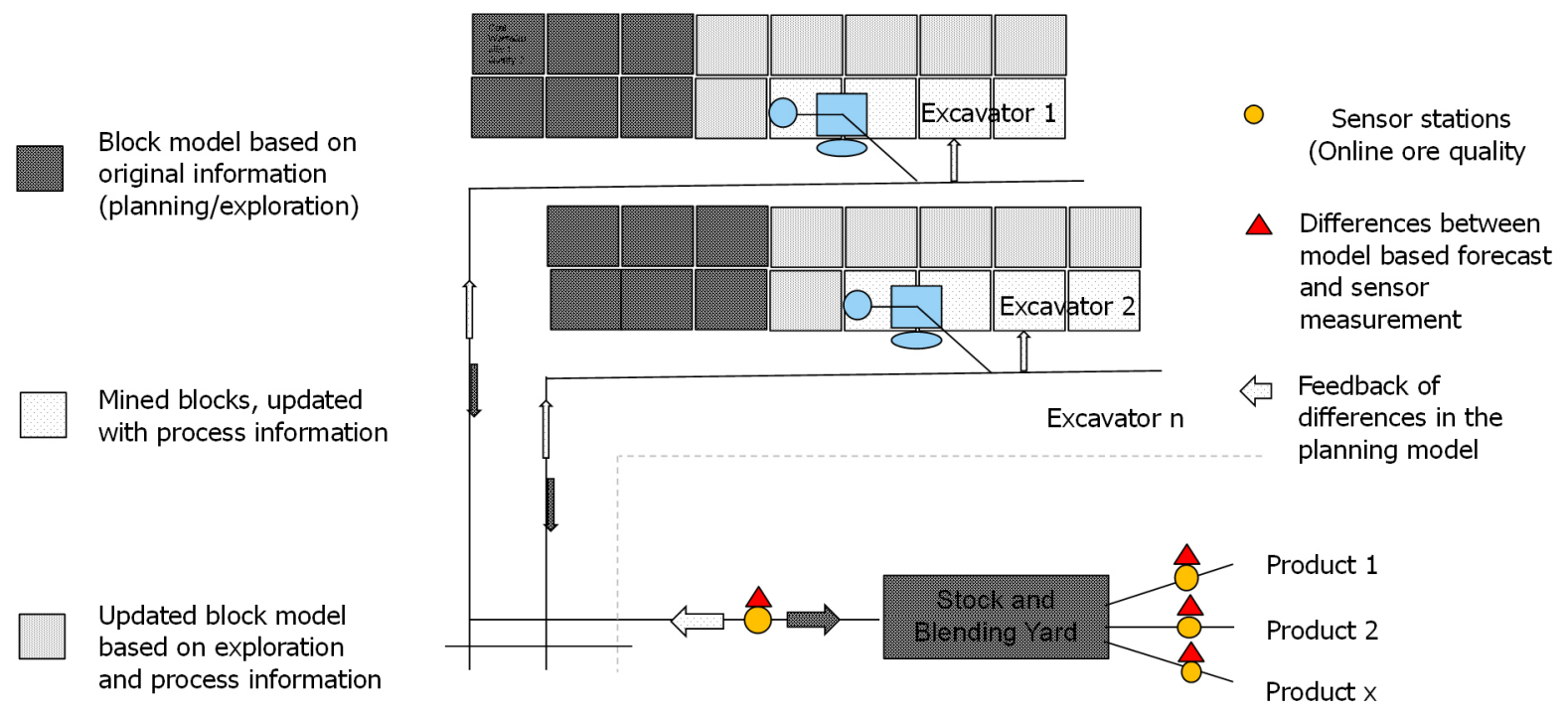

FIG 7 - Continuous mining system used in illustrative test case.

artificial sensor data represent a ten minute moving average (corresponding to about $250 \mathrm{t}$ production) and are composed of three components. Component one is the true block grade taken from the exhaustively known data set. Component two captures the volume variance relationship and corrects the smaller sensor measurement support of $250 \mathrm{t}$ to the mining block support of $5120 \mathrm{t}$ by adding the corresponding dispersion variance. The third component mimics the precision of the sensor. For this case study, the relative sensor error is varied between one per cent, five per cent and ten per cent.

\section{Evaluation measures}

The performance of the proposed KF approach was evaluated using two measures. The first measure is the mean square difference, or mean square error (MSE), related to the true block value. Here, the difference between estimated block value $z^{t+1}(x)$ and real block value $z(x)$ from the exhaustive data set is compared. The MSE is an empirical error measure and can be calculated according to:

$$
M S E=\frac{1}{N} \sum_{i=1}^{N}\left(z^{t+1}\left(x_{i}\right)-z\left(x_{i}\right)\right)^{2}
$$

The second measure is the theoretical block variance (BV), which can be calculated using Equation 3 for the KF and for the EnKF empirically based on the updated ensemble members.

\section{Results and discussion}

To evaluate the performance of different system configurations and sensor precisions, the following cases were investigated:

- operating only one excavator using KF (Case A)

- operating two excavators simultaneously using KF and EnKF (Case B)

- operating three excavators simultaneously using KF (Case C).

Table 1 summarises the parameters used in this example. In order to guarantee linear independency of rows in the production matrix $A$, a cyclic component was added to the extraction rates in the cases of two and three excavators. This cyclic behaviour is typical for continuous mining equipment and is observed in practice.

Figures 8 to 10 summarise the results for applying the KF to Cases A, B and C. Figure 11 shows the results for applying the
TABLE 1

Equipment parameter and model approach used.

\begin{tabular}{|l|c|c|c|c|}
\hline Case & $\begin{array}{c}\text { Extraction } \\
\text { rate }\end{array}$ & $\begin{array}{c}\text { Extraction } \\
\text { mode }\end{array}$ & $\begin{array}{c}\text { Sensor } \\
\text { precision }\end{array}$ & Method \\
\hline One excavator & $\mathrm{E}_{1}: 500 \mathrm{t} / \mathrm{h}$ & Constant & $1 \%, 5 \%, 10 \%$ & Kalman filter \\
\hline $\begin{array}{l}\text { Two } \\
\text { excavators }\end{array}$ & $\begin{array}{c}\mathrm{E}_{1}: 500 \mathrm{t} / \mathrm{h} \\
\mathrm{E}_{2}: 1000 \mathrm{t} / \mathrm{h}\end{array}$ & Cyclic & $1 \%, 5 \%, 10 \%$ & $\begin{array}{c}\text { Kalmin filter } \\
\text { and ensemble } \\
\text { Kalmin filter }\end{array}$ \\
\hline $\begin{array}{l}\text { Three } \\
\text { excavators }\end{array}$ & $\begin{array}{c}\mathrm{E}_{1}: 500 \mathrm{t} / \mathrm{h} \\
\mathrm{E}_{2}: 1000 \mathrm{t} / \mathrm{h} \\
\mathrm{E}_{3}: 2000 \mathrm{t} / \mathrm{h}\end{array}$ & Cyclic & $1 \%, 5 \%, 10 \%$ & Kalman filter \\
\hline
\end{tabular}

EnKF applied to Case B. Each figure shows both the MSE and $\mathrm{BV}$, which are separately calculated for already mined blocks, blocks directly adjacent to the mined blocks and blocks that are two block lengths away from mined blocks.

Figure 8 clearly demonstrates the ability of the KF-based approach to decrease the uncertainty of predicting block values by updating based on sensor data. Considering the MSE, the following observations can be made:

- For mined blocks, the uncertainty almost vanishes. This is expected because in the case of one excavator, the sensor measurements can be unambiguously tracked back to the source block. Residual uncertainties remain due to the sensor precision.

- Adjacent blocks are updated, resulting in a significant improvement compared to the prior model. For highprecision sensors, this improvement leads to a decrease of the MSE of about 40 per cent. This improvement is due to the positive covariance between two adjacent blocks. In addition, the sensor clearly influences the result.

- Blocks in the second next row are still updated. Due to the larger distance and the corresponding smaller covariance, the effect is less obvious compared to directly adjacent blocks. It is, however, still significant.

The comparison between the empirical MSE and the theoretical BV reveals that the theoretical error measure realistically reflects the true error. Observed BVs are quantitatively very similar to the MSE. Slight differences occur and are mainly due to the limited amount of blocks tested.

Figures 9 and 10 show the increased difficulty of the filter to track back the differences between the sensor measurements 


\section{Kalman-Filter: 1 Excavator}

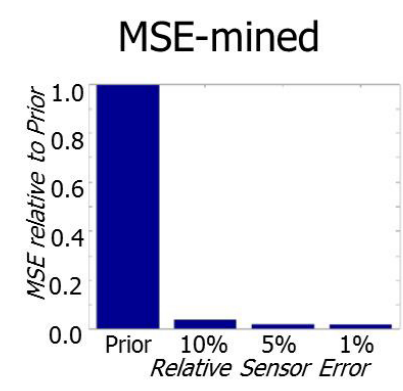

BV-mined

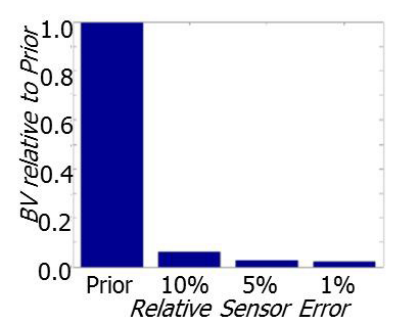

MSE- adjacent blocks MSE- 2 blocks away

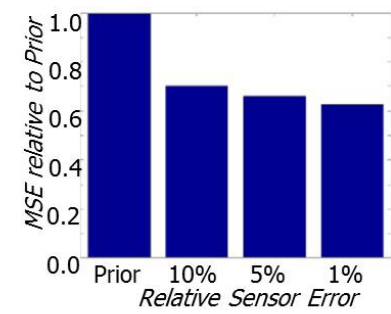

BV-adjacent blocks

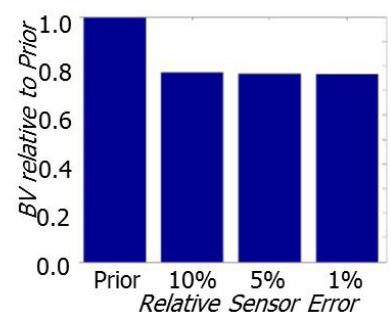

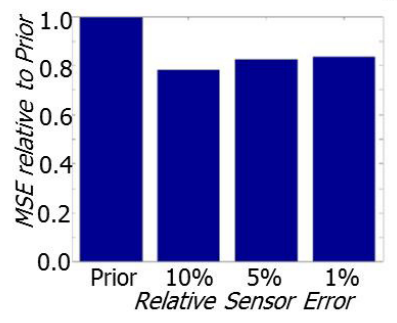

BV- 2 blocks away

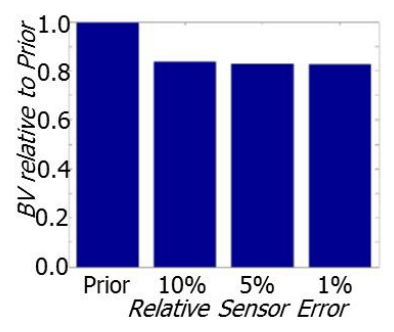

FIG 8 - Performance of the Kalman filter for updating the resource model in Case A.

\section{Kalman-Filter: 2 Excavators}

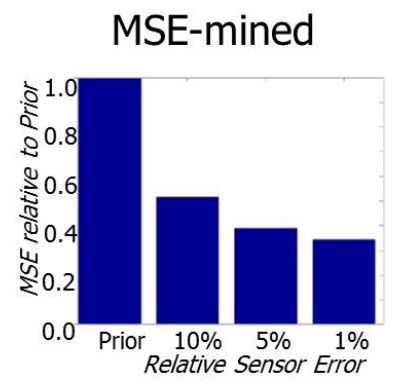

BV-mined

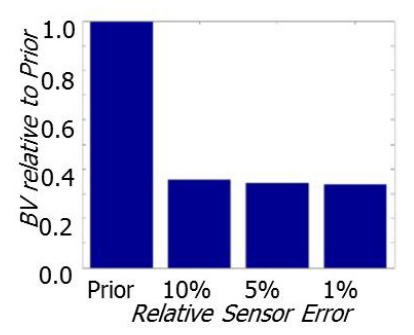

MSE- adjacent blocks MSE- 2 blocks away

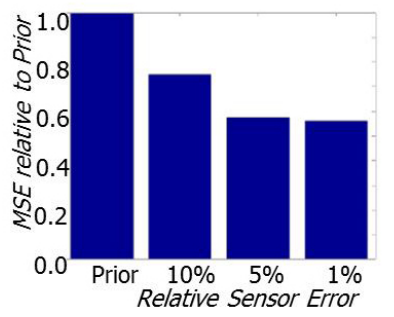

BV-adjacent blocks

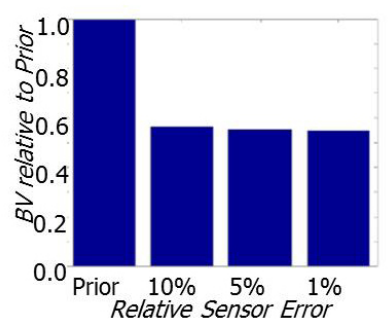

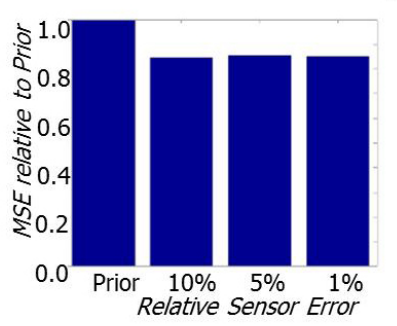

BV- 2 blocks away

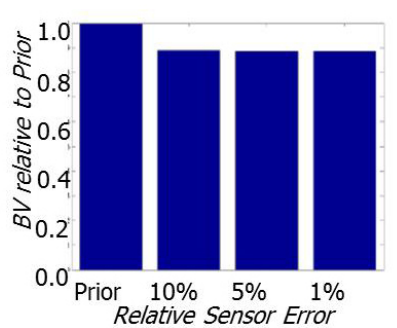

FIG 9 - Performance of the Kalman filter for updating the resource model in Case B.

and model-based predictions for combined material flow to the source blocks. The MSE and BV for mined blocks do not vanish completely; the remaining uncertainty can be interpreted as the limit of the filter for this specific application. It is expected that with increased sensor sampling (eg every two or five minutes instead of ten minutes), the performance can be improved. Nevertheless, there is still a significant improvement in prediction for directly adjacent blocks and the next row of blocks. Again, MSE and BV behave similarly. Differences are again due to local anomalies of grades in the tested blocks and locally varying sample data configuration (Figure 6).

Figure 11 shows the example of the EnKF applied to Case B. Results are very similar to Figure 9 and demonstrate the validity of using the EnKF. Due to the limited problem size, observations concerning computational efficiency cannot be regarded as representative.
Results demonstrate a significant level of improvement by incorporating sensor data, in this case about $15-40$ per cent relative compared to solely relying on exploration data. This improvement could be interpreted as a magnitude of frequency reduction of being out of spec for the delivery of coal to customers. The significant positive economic impact is obvious.

\section{CONCLUSIONS, VALUE OF REAL-TIME RESERVE MANAGEMENT AND FUTURE OUTLOOK}

The ability to incorporate online sensor data derived during the production process into resource/reserve models and the subsequent near real-time optimisation of short-term or operational decision variables promises a large potential for improvement in efficiency in any type of mining operation. 


\section{Kalman-Filter: 3 Excavators}

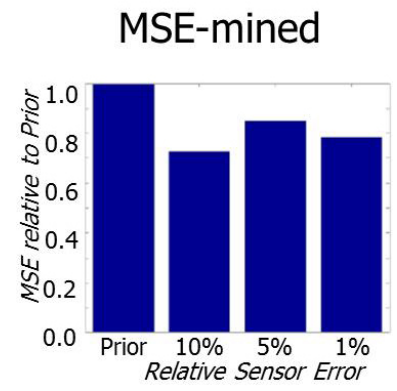

MSE- adjacent blocks MSE- 2 blocks away

BV-mined
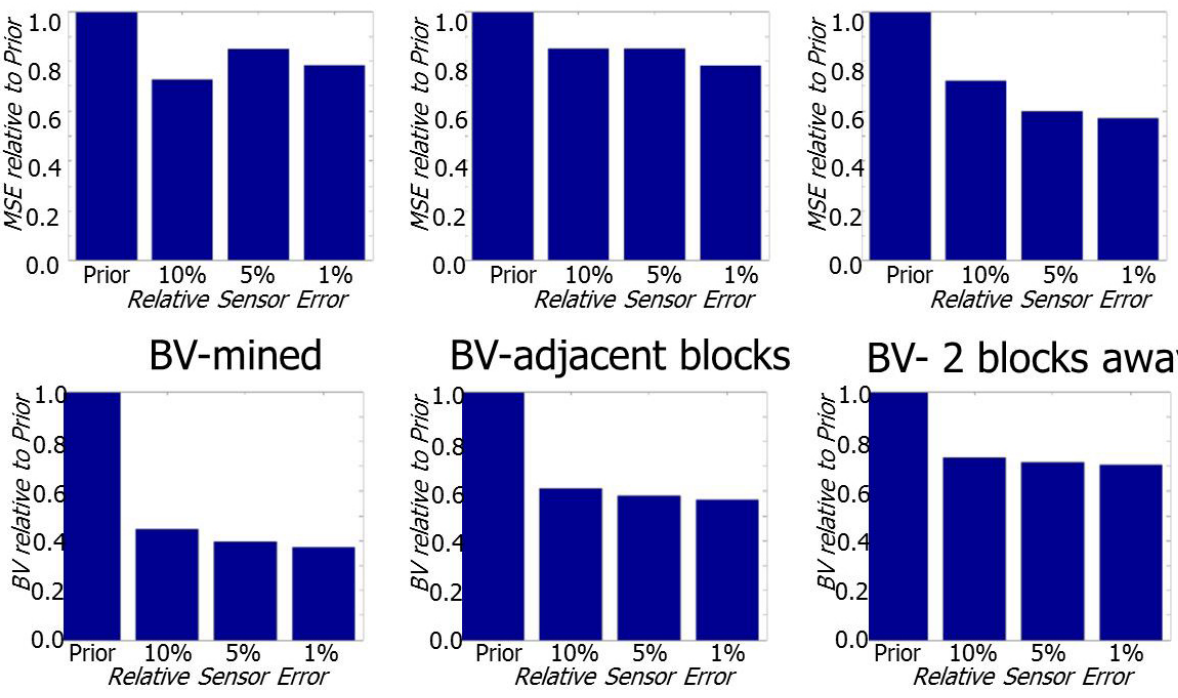

BV-adjacent blocks

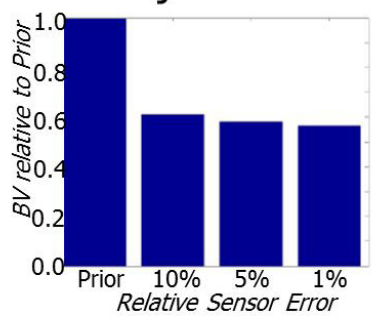

BV- 2 blocks away

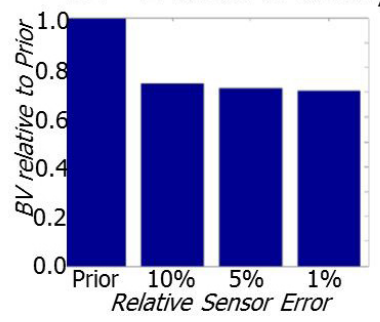

FIG 10 - Performance of the Kalman filter for updating the resource model in Case C.

\section{Ensemble Kalman-Filter: 2 Excavators}

\section{MSE-mined}

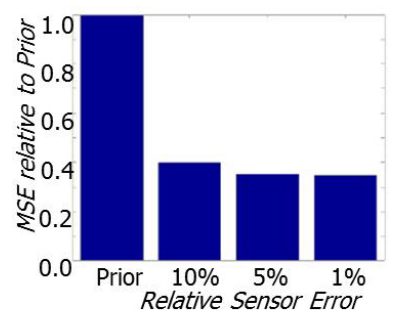

BV-mined

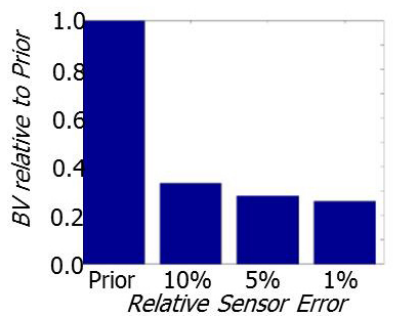

MSE- adjacent blocks MSE- 2 blocks away
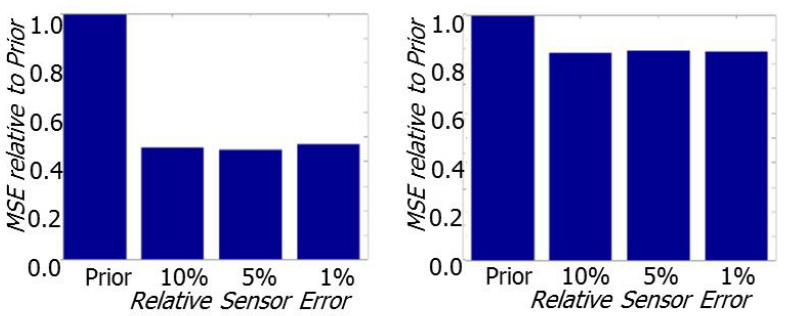

BV-adjacent blocks

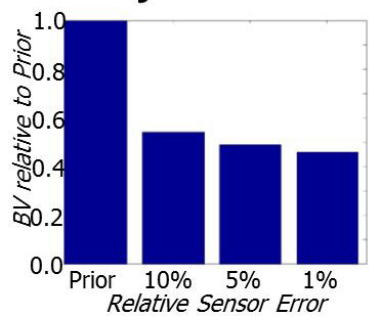

BV- 2 blocks away

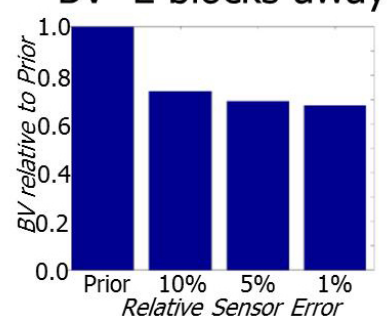

FIG 11 - Performance of the ensemble Kalman filter for updating the resource model in Case B.

This is especially the case when the variability of grades or quality parameters inherent in the deposit is medium to large. The economic effect of RTRM and mining process control can be quantified by a profit function J, which was adapted from (Engell, 2007):

$$
\begin{aligned}
& \Delta J=J\left(u_{\text {prior }}, d=0\right)-J\left(u_{\text {prior }}, d=d_{i}\right)+J\left(u_{\text {prior }}, d=d_{i}\right) \\
& -J\left(u_{\text {opt }}, d=d_{i}\right)+J\left(u_{\text {opt }}{ }^{\prime} d=d_{i}\right)-J\left(u_{\text {posterior }}, d=d_{i}\right)
\end{aligned}
$$

The first term is the loss due to the difference $d_{i}$ between actual and expected production targets, if decision variables $u_{\text {prior }}$ are fixed at their values based on the prior resource/ reserve model. The second term represents the difference between actual production targets achieved and the potential optimum resulting from an optimal adaptation of the decision variables $u_{\text {opt }}$ to the real conditions. The third term represents the compensation that is achieved by model updating and improved production decisions $u_{\text {posteriar }}$ It represents the residual uncertainty. This equation offers the means to evaluate if real-time optimisation is of any value. For example, if the first term in Equation 6 is much larger (in absolute value) than the second one, or if all terms are relatively small, then a variation of the decision variables offers no advantage. This can be the case at highly varying grades, where an adaption to real-time data corresponds to the adaption of noise. Realtime control aims to decrease the third term and should be designed at a timely resolution to reduce this difference to an anticipated level.

The presented framework of RTRM is a concept that is currently being further developed from a level of experimental proof-of-concept to a level of system prototype demonstration in an operational environment to prove industrial viability. The particular focus lies on the maturation of sensor technologies, overcoming limits of the feedback algorithms in terms of convergence as a function of system complexity and 
available data. In addition to grades or quality parameters, parameters that influence efficiency and recovery can also be integrated in the reserve model (eg by using GPS sensors and energy consumption recordings at excavators). This will require efficient data fusion algorithms. Simulation-based optimisation techniques will be developed further for efficient real-time optimisation of mine production control variables as new data become available.

With an implemented framework, further questions can be answered, such as: 'What is an efficient monitoring network for the system?' or 'What implications does the knowledge gained have on the long-term planning and necessary level of exploration?' The last question is particularly interesting as it investigates the utilisation of additional sensor data for mine planning and suggests that the level of 'traditional' exploration may be reduced in future. New exploration strategies for a 'self-learning mine' have to be developed that incorporate the time-effect of available information and maximise the use of it.

In the resource engineering section at the University of Technology in Delft, the Netherlands, current research projects are underway to mature this framework.

\section{REFERENCES}

Askari-Nasab, H, Torkamani, E, Badiozamani, M M and Tabesh, M, 2012. Alignment of short-term and operational plans using discrete event simulation, SME Annual Meeting Preprint 12-064.

Baafi, E and Ataeepour, M, 1996. Simulation of a truck-shovel system using arena, in Proceedings 26th Application of Computers and Operations Research in the Mineral Industry, pp 153-159.

Benndorf, J, in prep. A method for sequential teal-time updating of mineral resource models utilising online sensor data, Mathematical Geosciences.

Benndorf, J, 2013. Application of efficient methods of conditional simulation for optimising coal blending strategies in large continuous open pit mining operations, International Journal of Coal Geology, 112(2):141-153.

Benndorf, J, 2009. Evaluation of lignite deposits using conditional simulation in geostatistics, $221 \mathrm{p}$ published monograph at the Department of Geotechnik and Markscheidewesen of the TU Clausthal, Germany.

Benndorf, J and Dimitrakopoulos, R, 2013. Stochastic long-term production scheduling of iron ore deposits: integrating joint multi-element geological uncertainty, Journal of Mining Science, 49(1):68-81.

Bette, H, Noll, R, Müller, G, Jansen, H W, Nazikkol, C and Mittelstädt, H, 2005. Highspeed scanning LIBS at $1000 \mathrm{~Hz}$ with single pulse evaluation for the detection of inclusions in steel, J Laser Appl, 17(1):183-190.

Buxton, M and Benndorf, J, 2013. The use of sensor derived data in real time mine optimisation: a preliminary overview and assessment of techno-economic significance, paper presented at the 142nd SME Annual Meeting and Exhibit, preprint 13-038.

Chiles, J P and Delfiner, P, 2012. Geostatistics, Modelling Spatial Uncertainty, 734 p (John Wiley \& Sons: New York).

Death, D L, Cunningham, A P and Pollard, L J, 2008. Multi-element analysis of iron ore pellets by laser-induced breakdown spectroscopy and principal component regression, Spectrochima Acta, Part B, 63(1):763-769.

Dimitrakopoulos, R and Ramazan, S, 2009. Stochastic integer programming for optimising long-term production schedules of open pit mines: methods, application and value of stochastic solutions, IMM Transactions, Mining Technology, 117(4):155-160.

Engell, S, 2007. Feedback control for optimal process operation, Journal of Process Control, 17(3):203-219.
Escudero-Sanz, I, Ahlers, B and Bazalgette Courrèges-Lacoste, G, 2008. Optical design of a combined Raman-laser-inducedbreakdown-spectroscopy instrument for the European Space Agency ExoMars Mission, Optical Engineering, 47(3):033001033001.

Evensen, G, 2003. The ensemble Kalman filter: theoretical formulation and practical implementation, Ocean Dynamics, 53(4):343-367.

Goetz, A F H, Curtiss, B and Shiley, D A, 2009. Rapid gangue mineral concentration measurement over conveyors by NIR reflectance spectroscopy, Minerals Engineering, 22(1):490-499.

Gosavi, A, 2003. Simulation-Based Optimisation: Parametric Optimisation Techniques and Reinforcement Learning, 551 p (Springer: New York).

Hall, B E, 2000. Simulation modelling of mining systems, in Proceedings MassMin 2000, pp 83-96 (The Australasian Institute of Mining and Metallurgy: Melbourne).

Harris, P, Linton, P, Buxton, M, Pendock, N and Bars, R, 2010. Applications of near infrared core imaging, in Proceedings 3D Mineral Spectroscopy of the Earth's Skin - The 1st National Virtual Core Library Symposium.

Hoehse, M, Mory, D, Florek, S, Weritz, F, Gornushkin, I and Panne, U, 2009. A combined laser-induced breakdown and Raman spectroscopy Echelle system for elemental and molecular microanalysis, Spectrochimica Acta Part B: Atomic Spectroscopy, 64(11):1219-1227.

Isaaks, E H and Srivastava, R M, 1989. Applied Geostatistics, 561 p (Oxford University Press).

Jansen, J D, Douma, S D, Brouwer, D R, van den Hof, P M J, Bosgra, O H and Hemink, A W, 2009. Closed-loop reservoir management, paper SPE 119098 presented at the 2009 SPE Reservoir Simulation Symposium, 2-4 February, The Woodlands, USA.

Jewbali, A and Dimitrakopoulos, R, 2009. Stochastic mine planning? Example and value from integrating long - and short-term mine planning through simulated grade control, in Proceedings Orebody Modelling and Strategic Mine Planning 2009, pp 327-334 (The Australasian Institute of Mining and Metallurgy: Melbourne).

Jong, T P R, Dalmijn, W and Kattentid, H U R, 2003. Dual energy $\mathrm{x}$-ray transmission imaging for concentration and control, paper presented at IMPC 2003, Cape Town, South Africa.

Kalman, R E, 1960. A new approach to linear filtering and prediction problems, Journal of Basic Engineering, 82(1):35-45.

Kelton, W D and Law, A M, 2000. Simulation Modeling and Analysis, 760 p (McGraw Hill: Boston).

Ramazan, S and Dimitrakopoulos, R, 2004. Recent applications of operations research in open pit mining, SME Transactions, 316(1):73-78.

Salama, A, Nehring, M and Greberg, J, 2013. Operating value optimisation using simulation and mixed integer programming, International Journal of Mining, Reclamation and Environment, 28(1):25-46.

Scholze, P and Köhler, U, 2012. Complex control functions and management systems in the opencast mines of Vattenfall Europe Mining AG, World of Mining, GdmB, 31-39.

Schulze-Riegert, R and Shawket, G, 2007. Modern techniques of history matching, paper presented at 9th International Forum on Reservoir Simulation, Abu Dhabi.

Sharma, A K, Lucey, P G, Ghosh, M, Hubble, H W and Horton, K A, 2003. Stand-off Raman spectroscopic detection of minerals on planetary surfaces, Spectrochimica Acta Part A: Molecular and Biomolecular Spectroscopy, 59(1):2391-2407.

Sharma, A K, Misra, A K, Lucey, P G, Wiens, R C and Clegg, A M, 2007. Combined remote LIBS and Raman spectroscopy at $8.6 \mathrm{~m}$ of sulfur-containing minerals, and minerals coated with hematite or covered with basaltic dust, Spectrochimica Acta Part A, Molecular and Biomolecular Spectroscopy, 68(1):1036-1045. 
Shishvan, M S and Benndorf, J, 2013. Performance optimisation of complex continuous mining system using stochastic simulation, in Proceedings Fourth International Conference on Engineering Optimisation.

Sládková, D, Kapica, R and Vrubelm, H, 2011. Global navigation satellite system (GNSS) technology for automation of surface mining, International Journal of Mining, Reclamation and Environment, 25(3):284-294.

Subramaniam, G and Gosavi, G, 2007. Simulation-based optimisation for material dispatching in Vendor-Managed Inventory systems, Int J of Simulation and Process Modelling, 3(4):238-245.
Welsh, G and Bishop, G, 1997. An introduction to the Kalman Filter [online]. Available from: <http://www.cs.unc.edu/ welch/ media/pdf/kalman_intro.pdf> [Accessed: 24 July 2006].

Young Jung, J, Blau, G, Pekny, J F, Reklaitis, G V and Eversdyk, D, 2004. A simulation based optimisation approach to supply chain management under demand uncertainty, Computers and Chemical Engineering, 28(3):2987-2106.

Zimmer, B, 2012. Continuous mining equipment vs. complex geology challenges in mine planning, paper presented at 11th International Symposium of Continuous Surface Mining 2012, University of Miskolc. 
